INJE-TP-05-01

hep-th/0501023

\title{
Cosmic holographic bounds with UV and IR cutoffs
}

\author{
Yun Soo Myung* \\ Relativity Research Center and School of Computer Aided Science, Inje University \\ Gimhae 621-749, Korea
}

\begin{abstract}
We introduce the cosmic holographic bounds with two UV and IR cutoff scales, to deal with both the inflationary universe in the past and dark energy in the future. To describe quantum fluctuations of inflation on sub-horizon scales, we use the Bekenstein-Hawking energy bound. However, it is not justified that the D-bound is satisfied with the coarsegrained entropy. The Hubble bounds are introduced for classical fluctuations of inflation on super-horizon scales. It turns out that the Hubble entropy bound is satisfied with the entanglement entropy and the Hubble temperature bound leads to a condition for the slow-roll inflation. In order to describe the dark energy, we introduce the holographic energy density which is the one saturating the Bekenstein-Hawking energy bound for a weakly gravitating system. Here the UV (IR) cutoff is given by the Planck scale (future event horizon), respectively. As a result, we find the close connection between quantum and classical fluctuations of inflation, and dark energy.
\end{abstract}

\footnotetext{
${ }^{*}$ e-mail address: ysmyung@physics.inje.ac.kr
} 


\section{Introduction}

Supernova (SN Ia) observations [1] suggest that our universe is accelerating and the dark energy contributes $\Omega_{\mathrm{DE}} \simeq 0.60-0.70$ to the critical density of the present universe. Also the cosmic microwave background (CMB) observations [2] imply that the standard cosmology is given by the inflation [3] and flat FRW universe. It is generally accepted that curvature (scalar) perturbations produced during inflation are considered to be the origin of inhomogeneities necessary for explaining CMB anisotropies and large-scale structures.

The holographic principle is basically a statement about the number of fundamental degrees of freedom 4. In the case of scalar perturbations of inflation with the UV cutoff $\Lambda$, the holographic principle was used to derive the entropy bound for quantum fluctuations of inflation on sub-horizon scales [5]. It was shown that the indirect entropy bound leads to a constraint which states that the UV cutoff is nearly of the same order of the Planck scale. Also it was suggested that the D-bound is satisfied with the coarse-grained and entanglement entropies.

One of promising candidates for the dark energy is the cosmological constant. Cohen et al. [6] showed that in quantum field theory, the UV cutoff $\Lambda$ is related to the IR cutoff $L_{\Lambda}$ because of the limit set by forming a black hole. In other words, if $\rho_{\Lambda}$ is the quantum zeropoint energy density caused by the UV cutoff, the energy of the system with size $L_{\Lambda}$ should not exceed the mass of the system-size black hole: $L_{\Lambda}^{3} \rho_{\Lambda} \leq L_{\Lambda} M_{p}^{2}$ with the Planck mass of $M_{p}^{2}=1 / G$. The largest $L_{\Lambda}$ is then given by the one saturating this inequality and the holographic energy density takes the form of $\rho_{\Lambda} \sim\left(M_{p}^{2} / L_{\Lambda}\right)^{2}$. Recently the holographic energy density has been used to explain the dark energy widely [7, 8, 9, 10, 11, 12, 13. However, we mention that the future event horizon as the IR cutfof seems to be rather ad hoc chosen and it thus requires establishing a close connection between the holographic energy density and dark energy.

On the other hand, the implications of the cosmic holographic bounds have been investigated in the literature [14, 15, 16, 17, 18, 19. The cosmic holographic bounds are based on the holographic principle and the two Friedmann equations.

In this work we will examine how the cosmic holographic bounds with the UV and IR cutoffs could be used for describing fluctuations of inflation in the past and the dark energy in the future. Explicitly we use the Bekenstein-Hawking energy bound $E \leq E_{\mathrm{BH}}$ for a weakly gravitating system with $a \leq \sqrt{2-k} / H$, whereas for a strongly gravitating system

with $a \geq \sqrt{2-k} / H$, we use the Hubble entropy bound $S \leq S_{\mathrm{H}}$ and temperature bound $T \geq T_{\mathrm{H}}$. Especially, the energy bound is very useful for describing quantum fluctuations of inflation in the past and the dark energy in the future. In these cases, the UV cutoff is 
the Planck scale and the IR cutoff scale is the Hubble horizon for quantum fluctuations of inflation and the future event horizon for the dark energy. For classical fluctuations of inflation on super-horizon scales, we use the Hubble bounds to estimate the lower limit of its entropy and the upper limit of its temperature. Here the UV cutoff scale is also given by the Planck scale and the IR scale is the Hubble horizon on super-horizon scales.

The organization of this work is as follows. In section 2 we briefly review the cosmic holographic bounds: the Bekenstein-Hawking energy bound for a weakly gravitating system and the Hubble bounds for a strongly gravitating system. We apply the cosmic holographic bounds to the fluctuations of inflation: quantum fluctuations on sub-horizon scales and classical fluctuations on super-horizon scales in section 3. Section 4 is devoted to deriving the holographic energy density from the Bekenstein-Hawking energy bound. We show how the holographic energy density can explain the dark energy in Section 5. Finally we summarize our results in section 6 .

\section{Cosmic holographic bounds}

We briefly review the cosmic holographic bounds. Let us start an $(n+1)$-dimensional Friedmann-Robertson-Walker (FRW) metric

$$
d s^{2}=-d t^{2}+a(t)^{2}\left[\frac{d r^{2}}{1-k r^{2}}+r^{2} d \Omega_{n-1}^{2}\right],
$$

where $a$ is the scale factor of the universe and $d \Omega_{n-1}^{2}$ denotes the line element of an $(n-1)$-dimensional unit sphere. Here $k=-1,0,1$ represent that the universe is open, flat, closed, respectively. A cosmological evolution is determined by the two Friedmann equations

$$
\begin{aligned}
& H^{2}=\frac{16 \pi G_{n+1}}{n(n-1)} \frac{E}{V}-\frac{k}{a^{2}}, \\
& \dot{H}=-\frac{8 \pi G_{n+1}}{n-1}\left(\frac{E}{V}+p\right)+\frac{k}{a^{2}},
\end{aligned}
$$

where $H=\dot{a} / a$ represents the Hubble parameter and the overdot stands for derivative with respect to the cosmic time $t, E$ is the total energy of matter filling the universe, and $p$ is its pressure. $V$ is the volume of the universe, $V=a^{n} \sum_{k}^{n}$ with $\Sigma_{k}^{n}$ being the volume of an $n$-dimensional space with a curvature constant $k$, and $G_{n+1}$ is the Newton constant in $(n+1)$ dimensions. It denotes $G=1 / M_{p}^{2}$ for $(3+1)$-dimensional spacetime. Here we assume the equation of state: $p=\omega \rho, \rho=E / V$. First of all, we introduce two entropies 
for the holographic description of a universe [18]:

$$
S_{\mathrm{BV}}=\frac{2 \pi}{n} E a, \quad S_{\mathrm{BH}}=(n-1) \frac{V}{4 G_{n+1} a},
$$

where $S_{\mathrm{BV}}$ and $S_{\mathrm{BH}}$ are called the Bekenstein-Verlinde and Bekenstein-Hawking entropies, respectively. We identify $S_{\mathrm{BH}}$ with the Bekenstein-Hawking entropy of a universe-size black hole, which is close to the usual expression of $A / 4 G_{n+1}$. Then the first Friedmann equation can be rewritten as

$$
(H a)^{2}=2 \frac{S_{\mathrm{BV}}}{S_{\mathrm{BH}}}-k .
$$

We define a quantity $E_{\mathrm{BH}}$ which corresponds to energy needed to form a universe-sized black hole: $S_{\mathrm{BH}}=(n-1) V / 4 G_{n+1} a \equiv 2 \pi E_{\mathrm{BH}} a / n$. For $H a \leq \sqrt{2-k}$, one finds the Bekenstein-Hawking bound for a weakly self-gravitating system

$$
E \leq E_{\mathrm{BH}} \leftrightarrow S_{\mathrm{BV}} \leq S_{\mathrm{BH}}
$$

while for $H a \geq \sqrt{2-k}$, a holographic bound is given for a strongly self-gravitating system

$$
E \geq E_{\mathrm{BH}} \leftrightarrow S_{\mathrm{BV}} \geq S_{\mathrm{BH}} .
$$

The Bekenstein-Hawking bound is just a criterion on distinguishing between weakly and strongly gravitating system. Making use of the first Friedmann equation with $\mathrm{Ha}=$ $\sqrt{2-k}$, one finds that $S_{\mathrm{BV}}=S_{\mathrm{BH}} \leftrightarrow E=E_{\mathrm{BH}}$. For $a \leq \sqrt{2-k} / H$, we have to use the energy bound $E \leq E_{\mathrm{BH}}$ because its entropy bound $S_{\mathrm{BV}} \leq S_{\mathrm{BH}}$ is less important than the energy bound. This is because $S_{\mathrm{BV}}$ is not really an entropy but rather the energy. Also the role of $S_{\mathrm{BH}}$ is not to serve a bound on the total entropy, but rather on a sub-extensive component of the entropy. In this case, we may introduce the original Bekenstein bound on the entropy

$$
S \leq S_{\mathrm{BV}}
$$

This bound is restricted within narrow limits. Also there is no bound on the temperature of the system. Hence we use mainly the Bekenstein-Hawking energy bound for a weakly gravitating system with $H a \leq \sqrt{2-k}$.

For $H a \geq \sqrt{2-k}$, one has $S_{\mathrm{BV}} \geq S_{\mathrm{BH}}$ and the original Bekenstein bound in Eq.(17) should be replaced by an appropriate one. A good candidate is the Hubble entropy bound $S \leq S_{\mathrm{H}}$. Here $S_{\mathrm{H}}$ is proportional to the Bekenstein-Hawking entropy of a Hubble-size black hole $\left(H V_{\mathrm{H}} / 4 G_{n+1}\right)$ times the number of Hubble regions in the universe $\left(N_{\mathrm{H}}=\right.$ $\left.V / V_{\mathrm{H}} \geq 1\right)\left[18\right.$. That is, one finds that $S_{\mathrm{H}} \propto H V / 4 G_{n+1}$. According to the FischlerSusskind-Bousso (FSB) proposal, the entropy flow $S$ through a contracting sheet is less to 
equal to $A / 4 G_{n+1}\left(S \leq A / G_{n+1}\right)$, where $A$ is the area of the surface from which the light sheet originates. The infinitesimal form of this prescription leads to the Hubble entropy bound with the prefactor $(n-1)$.

In order to derive the Hubble bounds explicitly, the Friedmann equations (2) can be cast to the cosmological Cardy-Verlinde formula and cosmological Smarr formula, respectively

$$
\begin{aligned}
& S_{\mathrm{H}}=\frac{2 \pi a}{n} \sqrt{E_{\mathrm{BH}}\left(2 E-k E_{\mathrm{BH}}\right)}, \\
& k E_{\mathrm{BH}}=n\left(E+p V-T_{\mathrm{H}} S_{\mathrm{H}}\right),
\end{aligned}
$$

where the Hubble entropy $\left(S_{\mathrm{H}}\right)$ and temperature $\left(T_{\mathrm{H}}\right)$ are defined by

$$
S_{\mathrm{H}}=(n-1) \frac{H V}{4 G_{n+1}}, \quad T_{\mathrm{H}}=-\frac{\dot{H}}{2 \pi H} .
$$

These are useful quantities for describing a strongly gravitating phase with $H a \geq \sqrt{2-k}$ and are based on the holographic principle and Friedmann equations. Actually, Eq. (8) corresponds to another representations of the two Friedmann equations, which are expressed in terms of holographic quantities.

For a further study, we introduce a CFT-like radiation whose entropy and Casimir energy can be described by the Cardy-Verlinde formula and the Smarr formula, respectively

$$
\begin{aligned}
& S=\frac{2 \pi L}{n} \sqrt{E_{c}\left(2 E-E_{c}\right)}, \\
& E_{c}=n(E+p V-T S),
\end{aligned}
$$

where $S$ is the entropy of CFT-like radiation living on an $n$-dimensional space with size $L$ and $E$ is the total energy of CFT-like radiation. The first denotes the entropy-energy relation, while the second represents the relation between a non-extensive part of the total energy (Casimir energy) and thermodynamic quantities. Here $E_{c}$ and $T$ stand for the Casimir energy and the temperature of radiation with $\omega=1 / n$. The above equations correspond to thermodynamic relations for the CFT-radiation which are independent of the Friedmann equations. Suppose that the entropy of CFT-radiation in the FRW universe can be described by the Cardy-Verlinde formula. For general $k$, a bound on the Casimir energy $\left(E_{\mathrm{c}} \leq E_{\mathrm{BH}}\right)$ is equivalent to the Hubble entropy bound $S \leq S_{\mathrm{H}}$. It follows from Eqs.(8) and (10) that the Hubble bounds for entropy and temperature are given by 18

$$
S \leq S_{\mathrm{H}}, \quad T \geq T_{\mathrm{H}}, \text { for } H a \geq \sqrt{2-k}
$$

which shows two inequalities between geometric quantities $\left(S_{\mathrm{H}}, T_{\mathrm{H}}\right)$ and matter quantities $(S, T)$. The Hubble entropy bound can be saturated by the entropy of CFT-radiation 
filling the universe when its Casimir energy $E_{c}$ is large enough to form a universe-size black hole. That is, if $k E_{\mathrm{BH}}=E_{c}$, one has $S_{\mathrm{H}}=S$ and $T_{\mathrm{H}}=T$. If it happens, equations (8) and (10) coincide. This implies that the first Friedmann equation somehow knows the entropy formula for CFT-radiation filling the universe.

Hereafter we use the Bekenstein-Hawking energy bound $E \leq E_{\mathrm{BH}}$ for a weakly gravitating system with $a \leq \sqrt{2-k} / H$, whereas for a strongly gravitating system with $a \geq \sqrt{2-k} / H$, we use the Hubble bounds of $S \leq S_{\mathrm{H}}, T \geq T_{\mathrm{H}}$. Further the space dimensions is fixed to be $n=3$.

\section{Holographic bounds on the inflationary universe}

A holographic bound with the UV cutoff can be applied to describing the quantum fluctuations of inflation on sub-horizon scales [20]. The holographic principle provides a bound on the UV cutoff scale of the effective theory of inflation. Let us introduce the UV cutoff scale $\Lambda$ to parameterize our ignorance of physics beyond this scale. This means that the cutoff should be in physical momentum $(\wp<\Lambda)$. In this case the number of degrees of freedom depends on the UV cutoff. One usually assumes that $\Lambda \propto M_{p}$. On sub-horizon scales the energy density for scalar perturbations is expressed in terms of the UV cutoff $\Lambda$ and the Hubble horizon $d_{\mathrm{H}}=1 / H$ as [5]

$$
\rho_{\mathrm{qii}}=\frac{1}{32 \pi^{2}}\left(\Lambda / d_{\mathrm{H}}\right)^{2}=\frac{1}{32 \pi^{2}}(\Lambda H)^{2},
$$

which shows that for an effectively flat spacetime $(k=0)$, its equation of state is given by a radiation $p=\rho / 3$. In Section 5 , we will obtain the form of $\rho_{\mathrm{qfi}}$ from the BekensteinHawking temperature bound. On sub-horizon scales, its coarse-grained entropy is given by 21

$$
S_{\mathrm{cg}} \simeq \frac{\left(\Lambda d_{\mathrm{H}}\right)^{2}}{3 \pi}=\frac{1}{3 \pi}\left(\frac{\Lambda}{H}\right)^{2}
$$

which is the entropy for relativistic particles at early time before horizon crossing. Here there exist two candidates for the entropy bound. One is the direct entropy bound from the D-bound that appears when a relativistic matter is embedded in de Sitter space 22, 23. If one takes the Hubble horizon of $1 / H$ as the cosmological event horizon, then the increase of area is given by

$$
\Delta A=A_{\mathrm{f}}-A_{0}=\frac{\epsilon_{\mathrm{H}}}{2} A_{\mathrm{f}} .
$$

Here a Hubble slow-roll parameter $\epsilon_{\mathrm{H}}=-\dot{H} / H^{2}<1$ is in the region $0<\epsilon_{\mathrm{H}}<1$ for inflation, the initial horizon area $A_{0}$ for de Sitter space with the matter $\rho_{\mathrm{qf}}$, and 
the final horizon area $A_{\mathrm{f}}=4 \pi / H^{2}$ for de Sitter space after the matter is excited into the cosmological horizon. Then the D-bound implies that the entropy for the matter is limited by

$$
S \leq \frac{1}{4} \frac{\Delta A}{G}=\frac{\pi}{2} \frac{\epsilon_{\mathrm{H}} M_{p}^{2}}{H^{2}}=\frac{8 \pi^{2}}{3} \frac{\rho_{\mathrm{qfi}}}{H^{4}}
$$

Using the energy density in Eq.(12), this leads to the D-bound for quantum fluctuations of inflation $[5$

$$
S \leq \frac{1}{12} \frac{\Lambda^{2}}{H^{2}}
$$

Apparently the coarse-grained entropy in Eq.(13) violates the D-bound. Thus we cannot justify that the D-bound is satisfied with $S=S_{\mathrm{cg}}$ on sub-horizon scales. A relevant bound on quantum fluctuations of inflation seems to be the energy bound.

If one uses the potential slow-roll parameter $\epsilon_{\mathrm{V}}=1 / 2\left(V^{\prime} / V\right)^{2}$, one has the indirect entropy bound. In this case the change of entropy during one $e$-folding is limited by

$$
\Delta S \leq \frac{\pi}{2} \frac{\epsilon_{\mathrm{V}} M_{p}^{2}}{H^{2}}
$$

On the other hand, the amount of entropy that exists the horizon during one $e$-folding is given by $\Delta S=\Lambda^{2} / \pi H^{2}$. Then a bound on the UV cutoff takes the form

$$
\Lambda \leq \sqrt{8 \pi^{3} \epsilon_{\mathrm{V}}} M_{p}
$$

For an $e$-folding number $N=54\left(\sim 1 / \epsilon_{\mathrm{V}}\right)$, one finds $\Lambda \leq 2 M_{p}$. This means that the UV cutoff scale is nearly of the same order of the Planck scale $M_{p}$.

At late times after horizon crossing, the entropy is described by the entanglement entropy [5]

$$
S_{\text {ent }}=0.3\left(\Lambda d_{\mathrm{H}}\right)^{2}=0.3\left(\frac{\Lambda}{H}\right)^{2}
$$

which corresponds to the entropy for classical fluctuations of inflation outside the Hubble horizon (on super-horizon scales). This is nearly of the same order of the coarse-grained entropy and scales like the area of $d_{\mathrm{H}}^{2}=1 / H^{2}$. Now let us introduce the Hubble entropy bound in Eq.(11) because the system is in a strongly gravitating phase with $a \geq \sqrt{2} / H \sim$ $1 / H$. It is given by [18, 24]

$$
S \leq S_{\mathrm{H}} \sim N_{\mathrm{H}} \frac{M_{p}^{2}}{H^{2}}
$$

Taking $\Lambda \sim M_{p}$, the Hubble entropy bound can be rewritten as

$$
S \leq N_{\mathrm{H}} \frac{\Lambda^{2}}{H^{2}}
$$


It is evident from $a \geq 1 / H$ that $N_{\mathrm{H}} \geq 1$. Then one easily checks that the Hubble entropy bound is satisfied with the entanglement entropy $S_{\text {ent }}$.

We remark the Hubble temperature bound in Eq.(11). Expressing the Hubble temperature $T_{\mathrm{H}}$ in terms of $\epsilon_{\mathrm{H}}$ and the Gibbons-Hawking temperature $T_{\mathrm{GH}}=\frac{H}{2 \pi}$ [22], one finds the relation

$$
T_{\mathrm{H}}=\epsilon_{\mathrm{H}} T_{\mathrm{GH}} .
$$

A condition for an accelerating universe $\left(0<\epsilon_{\mathrm{H}}<1\right)$ leads to an inequality

$$
T_{\mathrm{H}}<T_{\mathrm{GH}} .
$$

This is another representation for inflation to occur, which is expressed in terms of the Hubble and Gibbons-Hawking temperatures. Now we introduce the matter temperature $T$ for classical fluctuations of inflation. The holographic temperature bound implies

$$
T \geq T_{\mathrm{H}} .
$$

We will show that this bound is valid for a slow-roll period of inflation. We recall that this bound is derived from the connection between the second Friedmann equation in Eq.(22) and CFT-like radiation. For a slow-roll period of inflation, the Hubble temperature is positive but less than the Gibbons-Hawking temperature $T_{\mathrm{GH}}$. For a de Sitter inflation, one has $T_{\mathrm{H}}=0$ and $T_{\mathrm{GH}}>0$. This means that at least the Hubble temperature is a limiting temperature to define the matter temperature $T$ as $T \geq 0$ from the Hubble temperature bound. For a de Sitter inflation, the holographic temperature bound leads to the definition of matter temperature: $T \geq 0$ exactly. In the case of $T_{\mathrm{GH}}=T_{\mathrm{H}}$, inflation comes to an end $\left(\epsilon_{\mathrm{H}}=1\right)$. In the slow-roll inflation there may be no cosmological horizon but one would still like to describe the approximate thermal state. Here we propose a stronger constraint on the matter temperature than the Hubble temperature bound

$$
T \geq T_{\mathrm{GH}}
$$

for classical fluctuations of inflation because the Hubble horizon plays a role of thermal heat bath with slowly varying Gibbons-Hawking temperature $T_{\mathrm{GH}}=H / 2 \pi$.

We find that the D-bound derived by analogy with de Sitter spacetime is not appropriate for describing quantum fluctuations of inflation with the UV cutoff $\Lambda$. This means that the entropy bound is not suitable for a weakly gravitating system. Instead, one expects that the energy bound will play an important role. On the other hand, the indirect entropy bound leads to a constraint on the UV cutoff scale which implies that $\Lambda \sim M_{p}$. Fortunately, we show that the Hubble entropy and temperature bounds are suitable for describing classical fluctuations of inflation on super-horizon scales. 


\section{Holographic energy bound}

Now we are in a position to ask how the cosmic holographic bounds use to describe the present accelerating universe. For an effective quantum field theory in a box of size $L_{\Lambda}$ with the UV cutoff $\Lambda$, its entropy scales extensively as

$$
S_{\Lambda} \sim L_{\Lambda}^{3} \Lambda^{3}
$$

where we choose the volume of the box as $V_{\Lambda}=4 \pi L_{\Lambda}^{3} / 3 \sim L_{\Lambda}^{3}$. This means that we start with the two independent UV and IR cutoffs. Before we proceed, we comment on the original Bekenstein bound of $S \leq S_{\mathrm{BV}}$ [4. Considering $S_{\mathrm{BV}}=2 \pi E_{\Lambda} L_{\Lambda} \sim\left(L_{\Lambda} \Lambda\right)^{4}$, this bound is automatically satisfied for an effective quantum field theory. Thus this bound does not provides any constraint for an effective theory.

Considering the thermodynamics of black holes, one postulated that the maximum entropy in the box of volume $V_{\Lambda}$ behaves non-extensively, growing only as the enclosed area $A_{\Lambda}$ of the box. If one limits the entropy to a holographic bound

$$
S_{\Lambda} \sim L_{\Lambda}^{3} \Lambda^{3} \leq S_{\mathrm{BH}} \equiv \frac{2}{3} \pi M_{p}^{2} L_{\Lambda}^{2} \sim\left(M_{p} L_{\Lambda}\right)^{2},
$$

the effective theory can describe the black hole. We call the above the Bekenstein bound to compare with the original Bekenstein bound of $S \leq S_{\mathrm{BV}}$. Here the IR cutoff cannot be chosen independently of the UV cutoff $\Lambda$. It scales like $L_{\Lambda} \sim \Lambda^{-3}$. On the other hand, Cohen et al. proposed another energy bound [6]

$$
E_{\Lambda} \sim L_{\Lambda}^{3} \Lambda^{4} \leq M_{\mathrm{S}} \sim L_{\Lambda} M_{p}^{2}
$$

where the IR cutoff scales as $L_{\Lambda} \sim \Lambda^{-2}$. This bound is more restrictive than the Bekenstein bound in Eq.(27). The two scales $\Lambda$ and $L_{\Lambda}$ are independent to each other in the beginning. To reconcile the breakdown of the quantum field theory to describe a black hole, one proposes a relationship between UV and IR cutoffs. Then we have an effective field theory with the IR cutoff which could describe a system including even black holes.

Now we explain the bound of Eq.(28) within our framework. Assuming that the present universe is in a weakly gravitating phase, we can reinterpret it in view of the Bekenstein-Hawking energy bound. From Eq.(15), one finds that the holographic bound is $E \sim L_{\Lambda}^{3} \Lambda^{4} \leq E_{\mathrm{BH}} \equiv 2 L_{\Lambda} M_{p}^{2} \sim L_{\Lambda} M_{p}^{2}$ with $a \sim L_{\Lambda}$. This leads to the energy bound in Eq.(28). Also this inequality can be derived from the Bekenstein-Hawking entropy bound of $S_{\mathrm{BV}} \leq S_{\mathrm{BH}}$. If $H a=\sqrt{2}$, one finds the saturation which means that $S_{\mathrm{BV}}=S_{\mathrm{BH}} \leftrightarrow E=E_{\mathrm{BH}}$. We remind the reader that $E_{\mathrm{BH}}$ is an energy to form a universesize black hole. The universe is in a weakly self-gravitating phase when its total energy 
$E$ is less than $E_{\mathrm{BH}}$, and in a strongly gravitating phase for $E>E_{\mathrm{BH}}$. Comparing with the Bekenstein bound in Eq.(27), the Bekenstein-Hawking bound comes out when taking both the Friedmann equation and holographic principle into account.

Consequently, the energy bound of Eq.(28) is nothing but the Bekenstein-Hawking energy bound for a weakly gravitating system. As is emphasized again, the energy is a rather important quantity for describing a weakly gravitating system than the entropy.

\section{Holographic dark energy}

In order to express the dark energy in terms of the holographic energy density, we take the largest $L_{\Lambda}$ as the one saturating the inequality of Eq.(28). Then we obtain a holographic energy density,

$$
\rho_{\Lambda}=\frac{3 c^{2}}{8 \pi}\left(\frac{M_{p}}{L_{\Lambda}}\right)^{2}
$$

with a numerical constant $3 c^{2}$. In other words, one uses the equality $E=E_{\mathrm{BH}}$ of the Bekenstein-Hawking bound to get the holographic energy density. The Planck scale comes from this energy bound naturally. The UV cutoff $\Lambda$ resolves into the IR cutoff $L_{\Lambda}$ and instead, the Planck scale $M_{p}$ plays a role of the UV cutoff.

We discuss the connection between Eq.(12) and Eq.(29). For a while, we neglect the prefactors. These are the same holographic form except replacing $\Lambda$ and $d_{\mathrm{H}}$ by $M_{p}$ and $L_{\Lambda}$. In the case of quantum fluctuations $\rho_{\mathrm{qfi}}$ of inflation on sub-horizon scales, the UV cutoff $\Lambda$ is determined to be the Planck scale $M_{p}$ by the indirect entropy bound. On the other hand, one can choose the IR cutoff $L_{\Lambda}$ to be the Hubble horizon because of $a \leq$ $\sqrt{2} / H \sim 1 / H$ on sub-horizon scales. Then, we obtain the energy density $\rho_{\text {qfi for quantum }}$ fluctuations of inflation from the holographic energy density $\rho_{\Lambda}$. Furthermore, we note that the coarse-grained, entanglement, and Hubble entropies take the same holographic form: $S \sim\left(M_{p} d_{\mathrm{H}}\right)^{2}$. From the Bekenstein entropy bound in Eq.(27), one finds the same form of holographic entropy: $S_{\Lambda} \sim\left(M_{p} L_{\Lambda}\right)^{2}$. A difference is between $d_{\mathrm{H}}=1 / H$ in the past and $L_{\Lambda}$ in the present. These show the close relationship between two weakly gravitating systems.

Here one has three possibilities for $L_{\Lambda}$ to describe the dark energy. If one chooses the IR cutoff as the size of our universe $\left(L_{\Lambda}=d_{\mathrm{H}}\right)$, the resulting energy density is comparable to the present dark energy [7]. Even though this holographic approach leads to the data, this description is incomplete because it fails to explain the present universe with $\omega \leq-0.78[8]$. Explicitly, the Friedmann equation including a matter of $\rho_{m}$ is then given by $\rho_{m}=3\left(1-c^{2}\right) M_{p}^{2} H^{2} / 8 \pi$, which shows that the equation of state for the dark 
energy is given by $\omega_{\mathrm{d}}=0$. However, an accelerating universe requires $\omega<-1 / 3$ and thus it is not the case. To resolve this situation, one is forced to introduce the particle horizon $L_{\Lambda}=R_{\mathrm{H}}=a \int_{0}^{t}(d t / a)$ which was used in the holographic description of cosmology by Fischler and Susskind [15]. The Friedmann equation of $H^{2}=8 \pi \rho_{\Lambda} / 3 M_{p}^{2}$ leads to an integral equation $H R_{H}=c$, which gives $\rho_{\Lambda} \sim a^{-2(1+1 / c)}$. Unfortunately it implies a decelerating universe with $\omega_{\mathrm{H}}=-1 / 3(1-2 / c)>-1 / 3$. In order to find an accelerating universe, we consider quantum fluctuations of inflation on sub-horizon scales. In this case, the Hubble horizon plays a role of the event horizon approximately [25]. Similarly we choose the future event horizon $R_{\mathrm{h}}=a \int_{t}^{\infty}(d t / a)$ as the IR cutoff $L_{\Lambda}$ [11]. Using the Friedmann equation of $H R_{\mathrm{h}}=c$, one finds $\rho_{\Lambda} \sim a^{-2(1-1 / c)}$ with $\omega_{\mathrm{h}}=-1 / 3(1+2 / c)<$ $-1 / 3$. For $c=1$, we recover a case of cosmological constant with $\omega_{\mathrm{h}}=-1$.

\section{Summary}

First of all we find that the D-bound derived by analogy with de Sitter spacetime is not appropriate for describing quantum fluctuations of inflation with the UV cutoff $\Lambda$. This means that the entropy bound is not suitable for a weakly gravitating system. Instead, one expects that the energy bound will play an important role. On the other hand, the indirect entropy bound leads to a constraint on the UV cutoff scale which implies that $\Lambda \sim M_{p}$. Fortunately, we show that the Hubble entropy and temperature bounds are suitable for describing classical fluctuations of inflation on super-horizon scales with $\Lambda \sim M_{p}$. This is because the classical fluctuations of inflation corresponds to a strongly gravitating system with $a \geq \sqrt{2} / H$.

Furthermore, the energy bound of Eq.(28) is nothing but the Bekenstein-Hawking energy bound for a weakly gravitating system. In this case the energy is a rather suitable quantity for describing a weakly gravitating system than the entropy. The holographic energy density $\rho_{\Lambda}$ is obtained from the one saturating the Bekenstein-Hawking energy bound. The energy density $\rho_{\mathrm{qfi}}$ for quantum fluctuations of inflation is closely related to the holographic energy density $\rho_{\Lambda}$ for the dark energy.

In order to describe the dark energy in terms of the holographic energy density, one has to choose the IR cutoff $L_{\Lambda}$. In this approach we don't know its equation of state exactly before solving the first Friedmann equation of $H L_{\Lambda}=c$. For quantum fluctuations of inflation on sub-horizon scales, we know that they behave a radiation with $\omega=1 / 3$. However, in the holographic approach to a weakly gravitating system, there exists information loss. This arises because one does not use the second Friedmann equation. As is shown in Eqs.(22) and (88), this contains information on the equation of state for a matter 
Table 1: Summary for three systems: quantum fluctuations of inflation (qfi) on subhorizon scales, classical fluctuations of inflation (cfi) on super-horizon scales, and dark energy (de) in the future. The two cutoff scales are introduced: the UV cutoff scale is the Planck scale $\left(\Lambda \sim M_{p}\right)$ for all three cases, but the IR cutoff scale depends on the system. Explicitly, $L_{\Lambda}=1 / H$, for qfi and cfi and $L_{\Lambda}=R_{\mathrm{h}}$ for de.

\begin{tabular}{|c|c|c|c|}
\hline & qfi & cfi & de \\
\hline type of system & weakly & strongly & weakly \\
\hline type of bound & $E \leq E_{\mathrm{BH}}$ & $S \leq S_{\mathrm{H}}, T \geq T_{\mathrm{H}}$ & $E \leq E_{\mathrm{BH}}$ \\
\hline energy density & $\rho_{\mathrm{qfi}}=\frac{1}{32 \pi^{2}}(\Lambda H)^{2}$ & $\rho_{\text {cfi }} \sim(\Lambda H)^{2}$ & $\rho_{\Lambda}=\frac{3 c^{2}}{8 \pi}\left(\frac{M_{p}}{R_{\mathrm{h}}}\right)^{2}$ \\
\hline entropy & $S_{c g}=\frac{1}{3 \pi}\left(\frac{\Lambda}{H}\right)^{2}$ & $S_{e n}=0.3\left(\frac{\Lambda}{H}\right)^{2}$ & $S_{\Lambda}=\left(M_{p} R_{\mathrm{h}}\right)^{3}$ \\
\hline equation of state & $p=\rho / 3$ & $p \sim \rho / 3$ & $p=-\rho$ for $c=1$ \\
\hline
\end{tabular}

partly. In the case of $k=0$, one obtains $(\rho+p) V=T_{\mathrm{H}} S_{\mathrm{H}}$ which shows that equation of state depends on the Hubble temperature $T_{\mathrm{H}}$. In the case of $T_{\mathrm{H}}=0$, one obtains $\omega=-1$ which is the de Sitter universe with a cosmological constant. In the case of $T_{\mathrm{H}} \neq 0$, one finds $p=-\rho+\left(S_{\mathrm{H}} / V\right) T_{\mathrm{H}}>-\rho$ which implies that $\omega>-1$. This means that the geometric quantities of $S_{\mathrm{H}}, T_{\mathrm{H}}$ and $V$ may determine the equation of state.

As is shown in Table 1 , the two combinations of the UV and IR cutoffs $\left(\Lambda / L_{\Lambda}, \Lambda L_{\Lambda}\right)$ determine all holographic energy densities and holographic entropies. In the case of $\rho_{\text {cfi }}$, even though its correct form is not known, one conjectures its form $\rho_{\text {cfi }} \sim(\Lambda H)^{2}$. As a result, there exist a close connection between three systems. Other approaches to the connection between inflation and dark energy appeared in ref. [26].

In conclusion the cosmic holographic bounds with the UV and IR cutoffs could describe weakly gravitating systems with $a \leq \sqrt{2} / H$. The energy bound is very useful for quantum fluctuations of inflation in the past and the dark energy in the future. In these cases, the UV cutoff is the Planck scale and the IR cutoff is the Hubble horizon for quantum fluctuations of inflation and the future event horizon for the dark energy. For classical fluctuations of inflation on super-horizon scales, we use the Hubble bounds to obtain the lower limit of its entropy and the upper limit of its temperature. Here the UV cutoff is also the Planck scale and the IR scale is the Hubble horizon on super-horizon scales.

\section{Acknowledgment}

This work was supported in part by KOSEF, Project Number: R02-2002-000-00028-0. 


\section{References}

[1] S. J. Perllmutter et al., Astrophys. J. 517, 565(1999) astro-ph/9812133]; A. G. Reiss et al., Astron. J. 116, 1009 (1998) astro-ph/9805201]; A. G. Reiss et al., Astrophys. J. 607, 665(2004) astro-ph/0402512;

[2] H. V. Peiris et al., Astrophys. J. Suppl. 148 (2003) 213 astro-ph/0302225; C. L. Bennett et al., Astrophys. J. Suppl. 148 (2003) 1 astro-ph/0302207; D. N. Spergel et al., Astrophys. J. Suppl. 148 (2003) 175 astro-ph/0302209.

[3] A. H. Guth, Phys. Rev. D 23, 347 (1981); A. D. Linde, Phys. Lett. B 108, 389 (1982); A. Albrecht and P. J. Steinhardt, Phys. Rev. Lett. 48, 1220 (1982).

[4] J. D. Bekenstein, Phys. Rev. D 23, 287 (1981).

[5] E. Keski-Vakkuri and M. S. Sloth, JCAP 0308, 001 (2003) hep-th/0306070.

[6] A. Cohen, D. Kaplan, and A. Nelson, Phys. Rev. Lett. 82, 4971 (1999) arXiv:hep-th/9803132.

[7] P. Horava and D. Minic, Phys. Rev. Lett. 85, 1610 (2000) arXiv:hep-th/0001145; S. Thomas, Phys. Rev. Lett. 89, 081301 (2002).

[8] S. D. Hsu, Phys. Lett. B 594,13 (2004) hep-th/0403052.

[9] P. Wang and X. Meng, Class. Quant. Grav. 22, 283(2005) astro-ph/0408495.

[10] R. Horvat, Phys. Rev. D 70, 087301 (2004) astro-ph/0404204.

[11] M. Li, Phys. Lett. B 603, 1 (2004) hep-th/0403127.

[12] Q-C. Huang and Y. Gong, JCAP 0408, 006 (2004) astro-ph/0403590; Y. Gong, Phys. Rev. D 70, 064029 (2004) hep-th/0404030; B. Wang, E. Abdalla and RuKeng Su, hep-th/0404057; S. Hsu and A. Zee, hep-th/0406142; P. F. Gonzalez-Diaz, hep-th/0411070; S. Nobbenhuis, gr-qc/0411093

[13] Y. S. Myung, hep-th/0412224.

[14] G. 't Hooft, gr-qc/9310026 L. Susskind, J. Math. Phys. 36, 6377 (1995) hep-th/9409089].

[15] W. Fischler and L. Susskind, hep-th/9806039. 
[16] R. Easther and D. A. Lowe, Phys. Rev. Lett. 82, 4967 (1999) hep-th/9902088; G. Veneziano, Phys. Lett. B 454, 22 (1999) hep-th/9902126; G. Veneziano, hep-th/9907012; R. Brustein and G. Veneziano, Phys. Rev. Lett. 84, 5695 (2000) hep-th/9912055; D. Bak and S. J. Rey, Class. Quant. Grav. 17, L83 (2000) hep-th/9902173; N. Kaloper and A. D. Linde, Phys. Rev. D 60, 103509 (1999) hep-th/9904120.

[17] R. Bousso, JHEP 9907, 004 (1999) hep-th/9905177; R. Bousso, JHEP 9906, 028 (1999) hep-th/9906022.

[18] E. Verlinde, hep-th/0008140.

[19] B. Wang, E. Abdalla, and T. Osada, Phys. Rev. Lett. 85, 5507 (2000) astro-ph/0006395; S. Nojiri and S. D. Odintsov, Int. J. Mod. Phys. A 16, 3273 (2001) hep-th/0011115; I. Savonije and E. Verlinde, Phys. Lett. B 507, 305 (2001) hep-th/0102042; S. Nojiri and S. D. Odintsov, Class. Quant. Grav. 18, 5227 (2001) hep-th/0103078; R. G. Cai, Y. S. Myung, and N. Ohta, Class. Quant. Grav. 18, 5429 (2001) hep-th/0105070; S. Nojiri, S. D. Odintsov, and S. Ogushi, Int. J. Mod. Phys. A 17, 4809 (2002) hep-th/0205187; R. G. Cai and Y. S. Myung, Phys. Rev. D 67, 124021 (2003) arXiv:hep-th/0210272; Y. S. Myung, Phys. Lett. B 578, 7 (2004) [arVix:hep-th/0306180; D. Youm, Phys. Lett. B 515, 170 (2001) arXiv:hep-th/0105093; M. R. Setare and R. Mansouri, Int. J. Mod. Phys. A 18, 4443 (2003) hep-th/0210252;

[20] N. Kaloper, M. Kleban, A. Lawrence, and S. Shenker, Phys. Rev. D 66, 123510 (2002) hep-th/0201158; U. H. Danielsson, Phys. Rev. D 66, 023511 (2002) hep-th/0203198; V. Bozza, M. Giovannini, and G. Veneziano JCAP 0305, 001 (2003) hep-th/0302184.

[21] M. Gasperini and M. Giovannini, Phys. Lett. B 301, 334 (1993) gr-qc/9301010.

[22] G. W. Gibbons and S. W. Hawking, Phys. Rev. D 15, 2738 (1977).

[23] V. Balasubramanian, J. de Boer, and D. Minic, Phys. Rev. D65 (2002) 123508 hep-th/0110108; Y. S. Myung, Mod. Phys. Lett. A16 (2001) 2353 hep-th/0110123; R. G. Cai, Y. S. Myung, and Y. Z. Zhang, Phys. Rev. D65 (2002) 084019 hep-th/0110234; A. M. Ghezelbash and R.B. Mann, JHEP 0201, 005 (2002) hep-th/0111217.

[24] C. J. Hogan, astro-ph/0406447. 
[25] R. Bousso, hep-th/0412197.

[26] K. Enqvist and M. S. Sloth, Phys. Rev. Lett. 93, 221302 (2004) hep-th/0406019; J. D. Bjorken, astro-ph/0404233; T. Padmanabhan, astro-ph/0411044. 